\title{
Characterization of Acid-Soluble Collagen from Skins of Surf Smelt (Hypomesus pretiosus japonicus Brevoort)
}

\author{
Takeshi Nagai $^{1 *}$, Nobutaka Suzuki ${ }^{2}$, Yasuhiro Tanoue ${ }^{3}$, Norihisa Kai ${ }^{3}$, Toshio Nagashima ${ }^{4}$ \\ ${ }^{1}$ Mimasaka University, Okayama, Japan; ${ }^{2}$ Nagoya Research Institute, Toyoake, Japan; ${ }^{3}$ National Fisheries University, Yamaguchi, \\ Japan; ${ }^{4}$ Tokyo University of Agriculture, Hokkaido, Japan. \\ Email: nagai-t@mimasaka.ac.jp, nagatakenagatake@yahoo.co.jp
}

Received July 23 ${ }^{\text {rd }}, 2010$; revised August $13^{\text {th }}, 2010$; accepted August $19^{\text {th }}, 2010$.

\begin{abstract}
Acid-soluble collagen was extracted from the skins of surf smelt and characterized. The yield of collagen was high about $24.0 \%$ on a dry weight basis. By SDS-polyacrylamide gel electrophoresis and CM-Toyopearl $650 \mathrm{M}$ column chromatography, this collagen is a heterotrimer with a chain composition of $\alpha 1 \alpha 2 \alpha 3$. The denaturation temperature was $32.5^{\circ} \mathrm{C}$, about $4.5^{\circ} \mathrm{C}$ lower than that from porcine skin. Attenuated total reflectance-fourier transform infrared analysis showed that the percentage of secondary structural components in this collagen were $11 \% \alpha$-helix, $34 \%$ $\beta$-sheet, $19 \% \beta$-turn, and $21 \%$ others. It suggests that the triple helical structure is present in the acid-soluble collagen from the skins of the surf smelt in comparison to that from the skin of porcine.
\end{abstract}

Keywords: Attenuated Total Reflectance-Fourier Transform Infrared (ATR-FTIR) Analysis, Collagen, Denaturation Temperature, Skin, Surf Smelt

\section{Introduction}

Collagen has been extracted from the skins of vertebrate species such as pig and calf of bovine for industrial applications as functional foods, cosmetics, and biomedical materials. However, these materials cannot be used for the extraction of collagen, due to the outbreak of bovine spongiform encephalopathy (BSE), transmissible spongiform encephalophathy (TSE), foot-and-mouth disease (FMD) [1], and avian influenza which resulted in anxiety among the users of collagen and collagen-derived products from these land animals. At present, alternatives to land animals as collagen sources are extremely desired. In recent years, we tried to extract and characterize the collagens from aquatic organisms because they have been reported not likely to be associated with infections such as BSE, TSE, and FMD, thus safe collagen samples can be obtained from aquatic organisms. A high yield of collagen could be obtained from aquatic organisms, although the physical and chemical properties of these collagens were different from those of land animals [2-14]. These results indicated that collagens from aquatic or- ganisms will have a potential to be important collagen sources for use in the food, cosmetic, and medical fields.

Surf smelt belong to the order Osmeridae which is a member of the Hypomesus family. The size of surf smelt (about $20 \mathrm{~cm}$ : body size; about $4 \%$ : the ratio of skin to body weight) is quite different from that of Japanese smelt (about $15 \mathrm{~cm}$ ) that is closely related to surf smelt. Surf smelt cannot live in freshwater, however, Japanese smelt can live in both freshwater and seawater. Surf smelt live not only around Hokkaido, Japan, but also in the Kuril Islands and in the Kamchatka Peninsula. The catch of surf smelt is about 11,000 t per year by inland water fisheries from Abashiri City of Hokkaido, Japan. The Japanese consume a wide range of fish species that include tunas, prawns, shellfishes, octopi, and squids. In particular, fried fish is widely eaten as tempura in Japan. Surf smelt is one of the fish that is eaten as tempura.

In the present study, we describe the isolation and physicochemical properties of acid-soluble collagen from the skins of surf smelt for use as an alternative to mammalian collagen in the food, cosmetic, and biomedical materials. 


\section{Materials and Methods}

\subsection{Sample}

Fresh surf smelt (Hypomesus pretiosus japonicus Brevoort) (50-65 g body weight and $15-18 \mathrm{~cm}$ body length) were caught in the port of Abashiri, Abashiri city, Hokkaido, Japan, and then transported in ice to our laboratory. These were stored at $-85^{\circ} \mathrm{C}$ until used.

\subsection{Preparation of Collagen from the Skins of the Surf Smelt}

All preparative procedures were carried out at $4{ }^{\circ} \mathrm{C}$ and were performed as described by Miller $[15,16]$. The frozen fish were thawed and the skins were descaled, trimmed of the residual fish meat, and cut out small pieces using a scalpel. The pieces $(0.5 \times 0.5 \mathrm{~cm})$ were homogenized for 3 days with 10 volumes of $10 \%$ ethanol to remove the fat by changing the solution twice a day. The homogenate was then squeezed using cheesecloth to remove any excessive ethanol, and the residue was washed with distilled water for 1 day by changing the solution twice a day. The matter was extracted for 2 days with $0.1 \mathrm{M} \mathrm{NaOH}$ in order to remove the noncollagenous proteins by changing the solution twice a day. The matter was squeezed using cheesecloth and then washed with distilled water for 2 days by changing the solution twice a day. After lyophilization, the dried matter was used for extraction of the acid-soluble collagen. The matter was treated for 2 days with 10 volumes of $0.5 \mathrm{M}$ acetic acid along with gentle stirring. The extract was centrifuged at $50,000 \times \mathrm{g}$ for $1 \mathrm{~h}$. The supernatants were pooled and salted out to isolate and purify the collagen by selective salt precipitation; the collagen was isolated by the addition of solid $\mathrm{NaCl}$ to a final concentration of $0.7 \mathrm{M} \mathrm{NaCl}$ in $0.5 \mathrm{M}$ acetic acid, followed by precipitation with $2.2 \mathrm{M}$ $\mathrm{NaCl}$ in $0.05 \mathrm{M}$ Tris-HCl buffer, $\mathrm{pH}$ 7.5. The purified collagen was obtained by centrifugation at $50,000 \mathrm{x} \mathrm{g}$ for $1 \mathrm{~h}$, and the precipitate was dissolved in a minimum volume of $0.5 \mathrm{M}$ acetic acid, dialyzed against $0.1 \mathrm{M}$ acetic acid for 2 days by changing the solution once a day, distilled water for 3 days by changing the solution twice a day, and then lyophilized (acid-soluble collagen).

Prior to the extraction of the collagen from bones, the bones (about $0.5 \times 0.5 \mathrm{~cm}^{2}$ ) were broken by a hammer, and then extracted with $0.1 \mathrm{M} \mathrm{NaOH}$ for 3 days to remove any noncollagenous proteins by changing the solution twice a day. The residue was washed with chilled distilled water and lyophilized. The collagen from the dried matter was also solubilized as described above.

\subsection{SDS-Polyacrylamide Gel Electrophoresis (SDS-PAGE) of Acid-Soluble Collagen from the Skins of the Surf Smelt}

SDS-PAGE was performed as previously described [8]. The collagen $(50 \mathrm{mg}$ protein) was dissolved in $0.5 \mathrm{M}$ Tris-HCl, pH 6.8, containing 2\% SDS, 25\% glycerol with 2-mercaptoethanol. After electrophoresis at $50 \mathrm{~mA}$ using $7.5 \%$ gel, the gel was stained with $0.25 \%$ Coomassie brilliant blue R-250 (Fluka Fine Chemical Co. Ltd., Tokyo, Japan) containing 25\% ethanol and $10 \%$ acetic acid and destained with 5\% methanol and 7.5\% acetic acid. Molecular weight markers [myosin (205 $\mathrm{kDa}), \beta$-galactosidase (116 kDa), phosphorylase B (97 $\mathrm{kDa})$, bovine serum albumin $(66 \mathrm{kDa})$, ovalbumin $(45$ $\mathrm{kDa})$, carbonic anhydrase $(29 \mathrm{kDa})$ ] were from the Sigma-Aldrich Co. (USA) and were used as marker proteins.

\subsection{Peptide Mapping of Acid-Soluble Collagen from the Skins of the Surf Smelt}

The collagen samples were dissolved in $0.1 \mathrm{M}$ sodium phosphate buffer ( $\mathrm{pH} 7.2$ ) containing $0.5 \%$ SDS to easily solubilized the collagen and bolied at $100^{\circ} \mathrm{C}$ for $5 \mathrm{~min}$. After cooling in ice, the denatured samples were digested at $37^{\circ} \mathrm{C}$ for $30 \mathrm{~min}$ using lysyl endopeptidase $(0.24 \mathrm{ami}-$ dase activity) from Achromobacter lyticus M497-1(EC 3.4.21.50; Wako Pure Chemical Industries, Ltd., Osaka, Japan). To stop the digestion, to the reaction mixture was added SDS to a final concentration of $2 \%$, and then boiled for $5 \mathrm{~min}$. The products were centrifuged at $30,000 \times \mathrm{g}$ for $5 \mathrm{~min}$, the supernatants were pooled and used as the sample solution for SDS-PAGE using 10\% gel. The molecular weight markers [myosin $(205 \mathrm{kDa})$, $\beta$-galactosidase (116 kDa), phosphorylase B (97 kDa), bovine serum albumin $(66 \mathrm{kDa})$, ovalbumin $(45 \mathrm{kDa})$, glyceraldehydes-3-phosphate dehydrogenase $(36 \mathrm{kDa})$, carbonic anhydrase $(29 \mathrm{kDa})$, bovine pancreas trypsinogen $(24 \mathrm{kDa})$, and soybean trypsin inbibitor $(20.1 \mathrm{kDa})]$ were from the Sigma-Aldrich Co. (USA) and were used as standards.

\subsection{Subunit Composition of Acid-Soluble Collagen from the Skins of the Surf Smelt}

Twenty milligrams of the collagen sample was dissolved in $20 \mathrm{mM}$ sodium acetate buffer ( $\mathrm{pH} 4.8$ ) containing $6 \mathrm{M}$ urea at $4^{\circ} \mathrm{C}$ for 1 day by continuous stirring. The viscous solution was denatured at $45^{\circ} \mathrm{C}$ for $30 \mathrm{~min}$, then centrifuged at $50,000 \mathrm{x} \mathrm{g}$ at $20^{\circ} \mathrm{C}$ for $1 \mathrm{~h}$. The supernatants were pooled and applied to a CM-Toyopearl $650 \mathrm{M}$ column $(1.0 \times 6.0 \mathrm{~cm})$ previously equilibrated with the same buffer. The elution was performed by a linear gradient of $0-0.15 \mathrm{M} \mathrm{NaCl}$ in the same buffer at the flow rate of $0.8 \mathrm{ml} / \mathrm{min}$. The subunit components were detected at $230 \mathrm{~nm}$ and the fractions indicated by the numbers were examined by SDS-PAGE using 7.5\% gel. 


\subsection{Denaturation Temperature of Acid-Soluble Collagen from the Skins of the Surf Smelt}

A Canon-Fenske type viscometer with an average shear gradient of $400 \mathrm{sec}^{-1}$ containing 5 milliliters of $0.03 \%$ collagen in $0.1 \mathrm{M}$ acetic acid was immersed in a water bath at temperatures of $20-50^{\circ} \mathrm{C}$. After incubation at each temperature for $30 \mathrm{~min}$, the viscosity of the collagen solution was measured and the fractional change was calculated as follows:

Relative viscosity $=\eta_{\mathrm{r}}=$ flow time of sample solution/flow time of $0.1 \mathrm{M}$ acetic acid

Specific viscosity $=\eta_{\mathrm{sp}}=\left(\eta-\eta_{0}\right) / \eta_{0}=\eta_{\mathrm{r}}-1$

The denaturation temperature was determined as the temperature causing a $50 \%$ decrease in viscosity. Each point is the mean of triplicate determinations.

\subsection{Amino Acid Analysis of Acid-Soluble Collagen from the Skins of the Surf Smelt}

An amino acid analysis was performed to clarify the relationship between the contents of the imino acids and the denaturation temperature of the collagen sample. The collagens were hydrolyzed under reduced pressure in $6 \mathrm{M}$ $\mathrm{HCl}$ at $110^{\circ} \mathrm{C}$ for $24 \mathrm{~h}$, and the hydrolysates were analyzed using a JASCO liquid-chromatography system by on-line precolumn derivatization with $o$-phthalaldehyde. This system consisted of a JASCO PU-2080 plus intelligent HPLC-pump, a JASCO FP-2020 plus intelligent fluorescence detector, a JASCO CO-2060 plus intelligent column thermostat, a JASCO DG-2083-53 3-line degasser, a JASCO LG-2080-02 ternary gradient unit, a JASCO AS-2057 plus intelligent sampler, and a JASCO CrestPak C18S $(\varphi 4.6 \times 150 \mathrm{~mm})$ reversed-phase column. The excitation and emission wavelengths were set at 345 and $455 \mathrm{~nm}$, respectively.

\subsection{Attenuated Total Reflectance-Fourier Transform Infrared (ATR-FTIR) Spectroscopy of Acid-Soluble Collagen from the Skins of the Surf Smelt}

The ATR-FTIR spectra were collected at $20^{\circ} \mathrm{C}$ and $40 \%$ relative humidity by coupling the ATR accessory (ATR PRO410-S: JASCO Co., Tokyo, Japan) to a JASCO (Tokyo, Japan) FT/IR-4100 type A instrument. The IR spectrometer bench was equipped with a globar source, a $\mathrm{KBr}$ bean splitter, and a triglycine sulfate detector. The ATR sampling device utilized a diamond internal reflection element embedded in a $\mathrm{ZnSe}$ support/focusing element in a single reflection configuration. The spectra were obtained over the range of $4000-650 \mathrm{~cm}^{-1}$ at a 4 $\mathrm{cm}^{-1}$ resolution. The resultant spectra were analyzed using an IR protein secondary structure analysis program (JASCO Co., Tokyo, Japan).

\section{Results and Discussion}

\subsection{Preparation of Acid-Soluble Collagen from the Skins of the Surf Smelt}

The skins from the surf smelt were treated with ethanol and followed by the removal of the noncollagenous constituents with $\mathrm{NaOH}$. As a result, the yield of the lyophilized matter was about $8.6 \%$ on a wet weight basis. Next, the matter was extracted with acetic acid. As a result, collagen was successfully solubilized and the yield was approximately $24.0 \%$ on a dry weight basis (about $2.1 \%$ on a raw weight basis). In recent years, a trial has been aggressively performed to extract the collagen from the aquatic organisms to utilize it in industry and the collagens obtained from these organisms have been used in the food, cosmetic, and medical fields. In fact, our groups have reported the yields of collagens from marine organisms as follows: fish skin (Japanese sea bass, 51.4\%, chub mackerel, $49.8 \%$, bullhead shark, $50.1 \%$, and ocellate puffer fish, 44.7\%) [7,10], purple sea urchin test (35.0\%) [11], fish bone (Japanese sea bass, 40.7\%, horse mackerel, $43.5 \%$, and ayu, 53.6\%) [12], edible jellyfish exumbrella (46.4\%) [4], rhizostomous jellyfish mesogloea (35.2\%) [5], Callistoctopus arakawai arm (62.9\%) [8], paper nautilus outer skin (50.0\%) [13], cuttlefish outer skin (35.0\%) [6], and common minke whale unesu [14], respectively. Recetly, some researchers reported the yields of fish skin collagen as follows: channel catfish acid-soluble (25.8\%) and pepsin-soluble collagens (38.4\%) [17], deep-sea redfish acid-solubilized (47.5\%) and pepsin-solubilized collagens $(92.2 \%)$ [18], and grass carp pepsin-soluble collagen (46.6\%) [19], respectively. On the other hand, the acid-soluble collagen from the bones of the surf smelt was only slightly extracted and the yield was only $0.8 \%$ on a dry weight basis.

\subsection{SDS-PAGE Patterns of Acid-Soluble Collagen from the Skins of the Surf Smelt}

Acid-soluble collagen from the skins of the surf smelt was measured by SDS-PAGE using $7.5 \%$ gel. The two distinct bonds were indicated in the stained gel in mobility in the $\alpha$ region; this collagen existed as trimers consisting of two distinct $\alpha$ chains, such as $\alpha 1$ and $\alpha 2$, although the existence of the $\alpha 3$ chain was not identified under this electrophoretic condition Figure 1. Moreover, a small amount of the $\beta$ chain was detected in this collagen. The same results were obtained in the collagen from the bones of the surf smelt. The positions of the chains of the collagen from the skins and bones of the surf smelt were similar to those of porcine skin Figure 1. These results indicate that acid-soluble collagens from the skins and bones of the surf smelt may have a chain composition of $(\alpha 1)_{2} \alpha 2$ heterotrimer are the type I collagen as a 
major component in mammalian collagen, such as porcine.

\subsection{Peptide Mapping of Acid-Soluble Collagen from the Skins of the Surf Smelt}

The collagen digested by lysyl endopeptidase (originally isolated from Achromobacter lyticus M497-1 specifically cleaves the peptides on the carboxy-terminal side of the lysine residues) was applied to SDS-PAGE using a 10\% gel to compare the patterns of the peptide fragment with porcine skin and the surf smelt bones. As a result, collagens from the skins and bones of the surf smelt were hydrolyzed to some extent by the enzyme with the nearly appearance of the peptide fragments with a molecular weight under about $80 \mathrm{kDa}$ : this indicates the digestion of subunit components having a molecular weight of about 110-130 kDa by lysyl endopeptidase Figure 2. On the other hand, the collagen from the porcine skin was not fully digested under this condition; the protein bands showing $\alpha$ and $\beta$ chains were detected, although there were some protein bands produced by digestion using the lysyl endopeptidase.

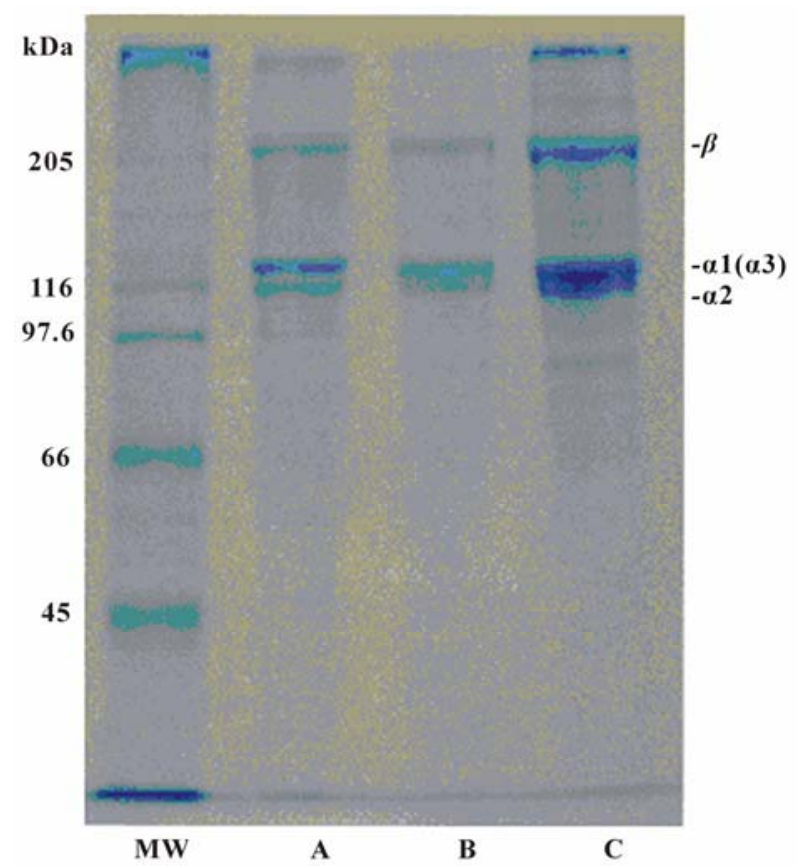

Figure 1. SDS-polyacrylamide gel electrophoresis of porcine

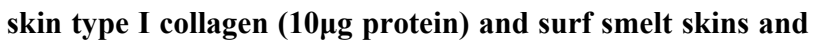
bone acid-soluble collagens $(10 \mu$ g protein) on $7.5 \%$ gels containing 3.5 M urea. (MW): high molecular marker: (A): acid-soluble collagen from the skins of the surf smelt; (B): acid-soluble collagen from bone of the surf smelt; $(\mathrm{C})$ : porcine skin collagen.

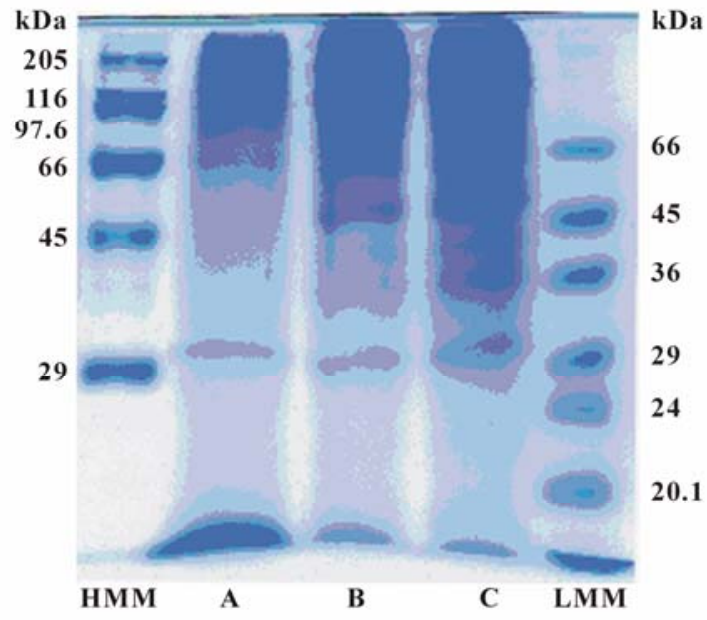

Figure 2. Peptide mapping of lysyl endopeptidase digests from collagen samples. (HMM): high molecular marker; (A) porcine skin collagen; (B) acid-soluble collagen from the skins of the surf smelt; $(C)$ acid-soluble collagen from bone of the surf smelt; (LMM): low molecular marker. The concentration of each collagen sample was used as $20 \mu \mathrm{g}$ protein.

\subsection{Subunit Composition of Acid-Soluble Collagen from the Skins of the Surf Smelt}

The collagen was denatured and then applied to a CMToyopearl $650 \mathrm{M}$ column to separate the subunit components of the acid-soluble collagen from the skins of the surf smelt. This was resolved by column chromatography into two peaks, one large and one small Figure 3. To identify each $\alpha$ chain, several chromatographic fractions as indicated by the numbers were analyzed by SDSPAGE. These results suggest that this collagen consists of three $\alpha$ chains. An $\alpha 1$ chain was found in fractions 5-8 as indicated by the numbers. An $\alpha 2$ chain was found in fractions 16-21 and the $\alpha 3$ chain in fractions 13-19 Figure 3. It suggested that this collagen is a heterotrimer with a chain composition of $\alpha 1 \alpha 2 \alpha 3$. Kimura et al. [20] examined the fish skin collagens and reported that the $\alpha 3$ chain was widely distributed in teleosts such as eel, sardine, chum salmon, rainbow trout, carp, anger, Alaska pollack, cod, halfbeak, common mackerel, tilapia, red barracuda, northern dab, and file fish. In a previous paper [20,21], it was reported that the $\alpha 3$ chain was detected in 14 fish species of 17 teleosts. On the other hand, Nagai et al. reported the subunit composition of collagen from aquatic organisms using the same techniques. The results were as follows: ocellate puffer fish skin [( $\left.\alpha 1)_{2} \alpha 2\right]$ [7], fish bone [Japanese sea bass: $(\alpha 1)_{2} \alpha 2$; horse mackerel: $(\alpha 1)_{3}$; ayu: $\alpha 1 \alpha 2 \alpha 3$ ] [12], fish scale from sardine, red sea bream, 

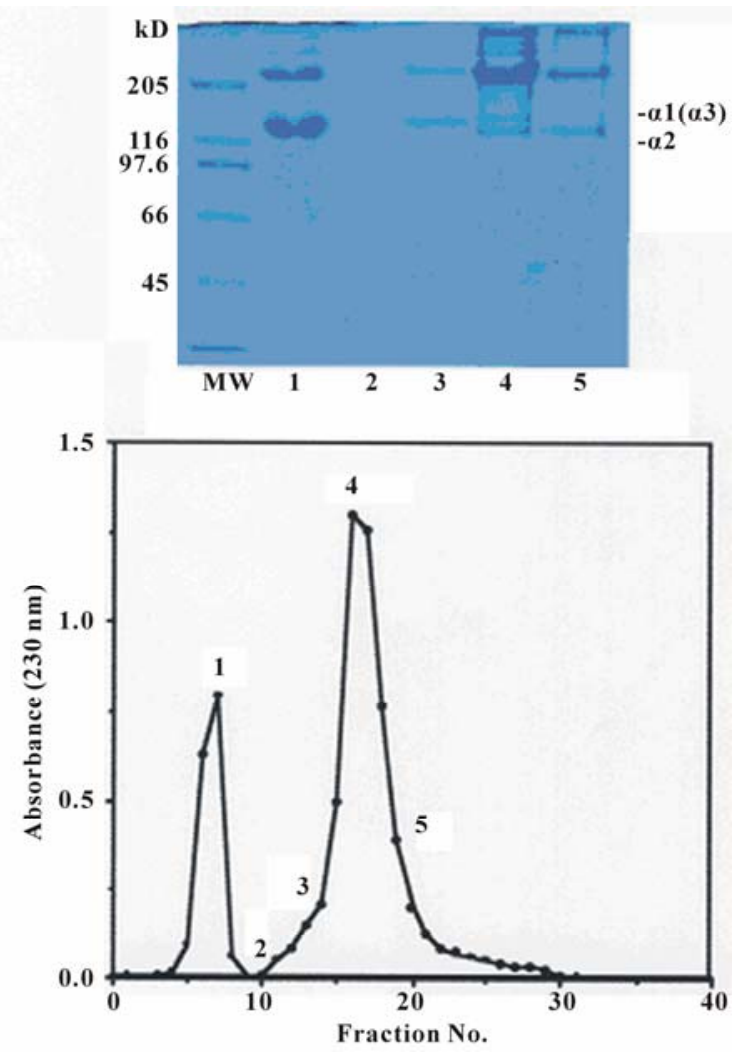

Figure 3. CM-Toyopearl $650 \mathrm{M}$ column chromatography of denatured acid-soluble collagen from the skins of the surf smelt. A $1.0 \times 6.0 \mathrm{~cm}$ column of CM-Toyopearl $650 \mathrm{M}$ was equilibrated with $0.02 \mathrm{M}$ sodium acetate buffer (pH 4.8) containing $6 \mathrm{M}$ urea, and maintained at $37^{\circ} \mathrm{C}$. The collagen sample (20.0 $\mathrm{mg}$ ) was dissolved in $5 \mathrm{ml}$ of the same buffer, denatured at $45^{\circ} \mathrm{C}$ for $30 \mathrm{~min}$, and then eluated from the column with a linear gradient of 0 to $0.15 \mathrm{M} \mathrm{NaCl}$ at a flow rate of $0.8 \mathrm{ml} / \mathrm{min}$. The fractions indicated by the numbers were examined by SDS-PAGE using $7.5 \%$ gel.

and Japanese sea bass $\left[(\alpha 1)_{2} \alpha 2\right]$ [9], caudul fin from Japanese sea bass $\left[(\alpha 1)_{3}\right][2]$, purple sea urchin test $\left[(\alpha 1)_{2} \alpha 2\right]$ [11], cuttlefish [( $\left.\left.\alpha 1\right)_{2} \alpha 2\right]$ [6], diamondback squid $(\alpha 1 \alpha 2 \alpha 3)$ [3], paper nautilus outer skin and $C$. arakawai arm $(\alpha 1 \alpha 2 \alpha 3)$ [8]; edible jellyfish exumbrella $(\alpha 1 \alpha 2 \alpha 3)$ [4], rhizostomous jellyfish mesogloea $(\alpha 1 \alpha 2 \alpha 3 \alpha 4)$ [5], and common minke whale unesu $\left[(\alpha 1)_{2} \alpha 2\right]$ [14]. That is, it was obvious that the $\alpha 3$ chain was significantly present in the skins from aquatic organisms such as jellyfishes, cuttlefishes, and octopi.

\subsection{Denaturation Temperature of Acid-Soluble Collagen from the Skins of the Surf Smelt}

The denaturation temperature of the acid-soluble collagen from the skins of the surf smelt was calculated from the thermal denaturation curve. For comparison, the de- naturation temperature of collagen from porcine skin was similarly measured. It was calculated that the denaturation temperature of the acid-soluble collagen from the skins of the surf smelt was about $32.5^{\circ} \mathrm{C}$ Figure 4. This was about $4.5^{\circ} \mathrm{C}$ lower than that of the collagen from porcine skin $\left(37.0^{\circ} \mathrm{C}\right)$. This value was fairly higher than those from other aquatic organisms reported by Nagai et al. as follows: ocellate puffer fish skin $\left(28.0^{\circ} \mathrm{C}\right)$ [7], fish scale from sardine $\left(28.5^{\circ} \mathrm{C}\right)$, red sea bream $\left(28.0^{\circ} \mathrm{C}\right)$, Japanese sea bass $\left(28.0^{\circ} \mathrm{C}\right)$ [9], caudul fin from Japanese sea bass $\left(28.0^{\circ} \mathrm{C}\right)[2]$, purple sea urchin test $\left(28.0^{\circ} \mathrm{C}\right)$ [11], cuttlefish $\left(27.0^{\circ} \mathrm{C}\right)[6]$, diamondback squid $\left(27.5^{\circ} \mathrm{C}\right)[3]$, paper nautilus outer skin $\left(27.0^{\circ} \mathrm{C}\right)$ [13], C. arakawai arm $\left(28.0^{\circ} \mathrm{C}\right)$ [8], edible jellyfish exumbrella $\left(26.0^{\circ} \mathrm{C}\right)$ [4],

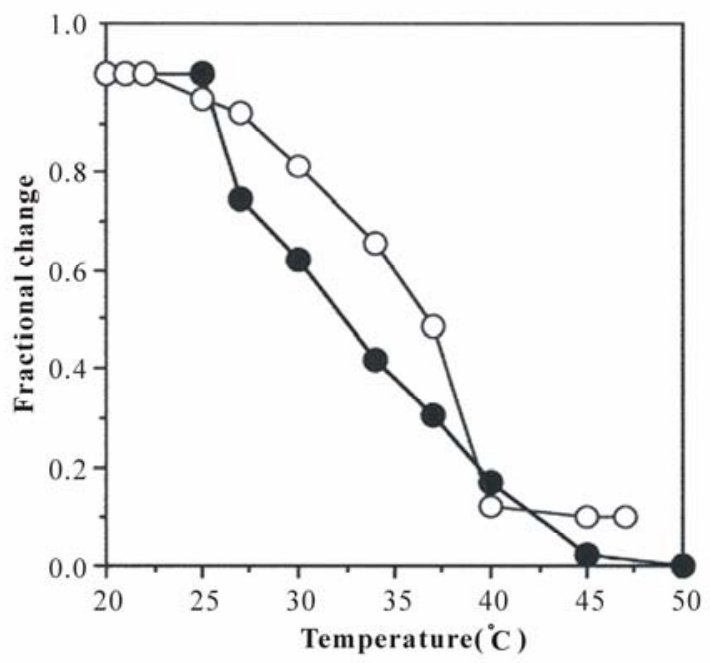

Figure 4. Thermal denaturation curves of acid-soluble collagen from the skins of the surf smelt and collagen from the skins of porcine. The denaturation temperature was measured by viscosity in $0.1 \mathrm{M}$ acetic acid. The incubation time at each temperature was $30 \mathrm{~min}$. Collagen concentration $0.03 \%$; $(\circ)$ : porcine skin collagen; $(\bullet)$ : acid-soluble collagen from the skins of the surf smelt.

rhizostomous jellyfish mesogloea $\left(28.8^{\circ} \mathrm{C}\right)$ [5], and common minke whale unesu $\left(31.5^{\circ} \mathrm{C}\right)$ [14]. It is well known that the denaturation temperature can be correlated with the environmental and body temperatures [22]. However, the present result was not applicable to those established theories. The reason for the high denaturation temperature of the collagen from the surf smelt is due to the difference in the hydroxylation ratio of proline, which is highly correlated to the thermal stability [23].

\subsection{Amino Acid Composition of Acid-Soluble Collagen from the Skins of the Surf Smelt}

The amino acid composition expressed as residues per 
1000 total residues, is shown in Table 1. It shows that glycine was the most abundant amino acid in the acidsoluble collagen from the skins of the surf smelt and accounted for more than about one third (343 residues) among the total amino acids in this collagen. There were relatively higher contents of alanine (123 residues), proline (108 residues), and glutamic acid (74 residues), decreasing in that order. Tyrosine (3 residues), histidine (6 residues), hydroxylysine (7 residues), isoleucine (10 residues), methionine (14 residues), and phenylalanine (15 residues) were low. The imino acid, such as proline and hydroxyproine, were also detected, 172 residues: the total contents of the imino acid in this collagen were $17.2 \%$. In previous papers, we reported the total contents of the imino acid in the collagen samples from aquatic organisms. As a result, the contents were as follows: fish scale (17.0-19.7\%) [7,9], fish fin (19.3\%) [2], octopus and cuttlefish skin (17.8-18.8\%) [3,6,8], sea urchin test (17.9\%) [11], jellyfish exumbrella (12.2\%) [4], and common minke whale unesu (19.9\%) [14], respectively. In recent years, other researchers reported the total contents of the imino acid in fish skin collagens as about $16.0-19.3 \%[17,19,24,25]$. The degree of hydroxylation of the proline residues in the acid-soluble collagen from the skins of the surf smelt was calculated. It is well known that hydroxyproline was derived from proline by post-translational hydroxylation mediated by prolylhydroxylase, and the degree of hydroxylation was associ-

Table 1. Amino acid composition of acid-soluble Collagen from the skins of the surf smelt.

\begin{tabular}{cc}
\hline Amino acid & residues/1000 \\
\hline Hydroxyproline & 64 \\
Hydroxylysine & 7 \\
Aspartic acid & 47 \\
Threonine & 22 \\
Serine & 44 \\
Glutamic acid & 74 \\
Proline & 108 \\
Glycine & 343 \\
Alanine & 123 \\
Valine & 20 \\
Methionine & 14 \\
Isoleucine & 10 \\
Leucine & 21 \\
Tyrosine & 3 \\
Phenylalanine & 15 \\
Lysine & 26 \\
Histidine & 6 \\
Arginine & 53 \\
Total & 1000 \\
\hline
\end{tabular}

ated with the high denaturation temperature of the collagen sample. The degree of hydroxylation of the proline residues in this collagen was calculated to be about $37.2 \%$. The previous papers showed the degree of hydroxylation of the proline residues in the collagen from aquatic organisms. As a result, the degrees of hydroxylation in collagens from fish scale [9] and fin [2], octopus [8] and cuttlefish $[3,6]$ skins, sea urchin test [11], and common minke whale unesu [14] were high, but the degrees of hydroxylation in collagens from the fish skins were low $[18,19,24,25]$. It was suggested that the reason for not only the lower denaturation temperatures in the collagen samples from aquatic organisms is the extent of hydroxylation of imino acids, but also the lower content of the imino acid.

\subsection{ATR-FTIR Spectroscopy Analysis of Acid-Soluble Collagen from the Skins of the Surf Smelt}

The ATR-FTIR spectrum of the collagen from the skins of the surf smelt is shown in Figure 5. The amide A band position was detected at $3306 \mathrm{~cm}^{-1}$, and it associated with the N-H stretching vibration. This indicates the existence of hydrogen bonds in the collagen sample. It is known that a free $\mathrm{N}-\mathrm{H}$ stretching vibration occurs in the range of 3400 to $3440 \mathrm{~cm}^{-1}$, and when the NH group of a peptide is involved in a hydrogen bond, the position is shifted to lower frequencies [26]. In other words, it suggested that more $\mathrm{NH}$ groups of the acid-soluble collagen from the skins of the surf smelt were involved in the hydrogen bonding. Next, the amide $\mathrm{B}$ band, related to the asymmetrical stretch of $\mathrm{CH}_{2}$, was found at $2964 \mathrm{~cm}^{-1}$ in this collagen. The amide I band of this collagen was detected at $1640 \mathrm{~cm}^{-1}$. The amide I band with its characteristic frequencies $\left(1600-1700 \mathrm{~cm}^{-1}\right)$, mainly associated with the stretching vibrations of the carbonyl group along the polypeptide backbone [27], is a sensitive marker of the peptide secondary structure [28]. The amide II and III bands were found at 1541 and $1235 \mathrm{~cm}^{-1}$, respectively. These indicated $\mathrm{N}-\mathrm{H}$ bending vibrations and $\mathrm{C}-\mathrm{H}$ stretching [27]. It suggested helical arrangements in the acid-soluble collagen from the skins of the surf smelt. Sarver and Krueger [29] investigated the protein secondary structure using FTIR and analyzed the resulting spectra to construct a database. To ensure that the extracted collagen was still in the native form using the secondary structure data, the theoretical value of the $\alpha$-helix, $\beta$-sheet, and $\beta$-turn in the native collagen should be provided and a comparison should be made. As a result, the percentage of these components in the acid- soluble collagen from the skins of the surf smelt was $11 \% \alpha$-helix, $34 \% \beta$-sheet, $19 \% \beta$-turn, and $21 \%$ others. 


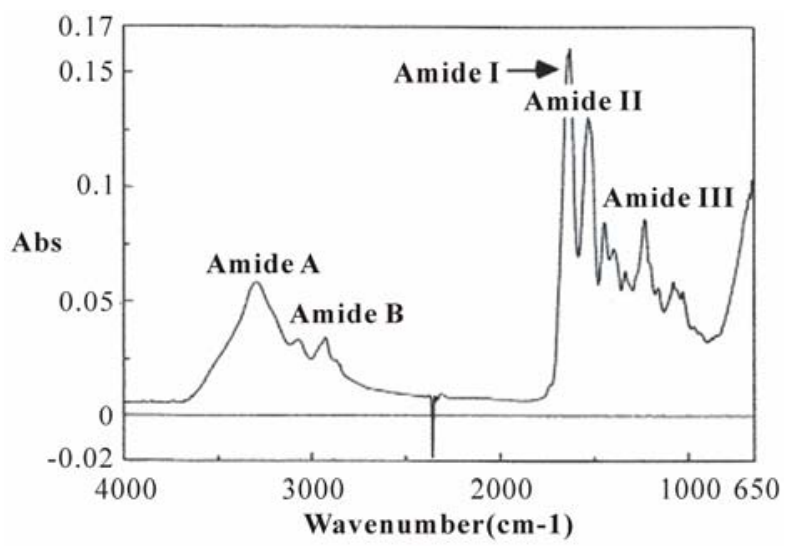

Figure 5. Fourier transform infrared spectra of acid-soluble collagen from the skins of the surf smelt.

For comparison, those of collagen from porcine skin were also calculated as follows: $9 \% \alpha$-helix, $51 \% \beta$-sheet, $13 \% \beta$-turn, and $15 \%$ others. Moreover, those of collagen from common minke whale unesu were as follows: $8 \%$ $\alpha$-helix, $50 \% \beta$-sheet, $15 \% \beta$-turn, and $17 \%$ others [14]. It suggests that the triple helical structure is present in the acid-soluble collagen from the skins of the surf smelt.

\section{Conclusions}

Acid-soluble collagen was extracted from the skins of the surf smelt and characterized. The denaturation temperature of this collagen was high in comparison to those of other aquatic organisms. By ATR-FTIR analysis using an IR protein secondary structure analysis program developed by the JASCO Co. (Tokyo, Japan), the percentage of the secondary structural components of the collagen sample was determined. Applying this technique will have the potential to obtain new knowledge about many species of collagens.

\section{REFERENCES}

[1] A. Jongjareonrak, S. Benjakul, W. Visessanguan, T. Nagai and M. Tanaka, "Isolation and Characterization of Acid and Pepsin-Solubilised Collagens from the Skin of Brownstripe Red Snapper (Lutjanus vitta)," Food Chemistry, Vol. 93, No. 3, 2005, pp. 475-484.

[2] T. Nagai, "Characterization of Collagen from Japanese Sea Bass Caudal Fin as Waste Material," European Food Research and Technology, Vol. 218, No. 5, 2004, pp. 424-427.

[3] T. Nagai, "Collagen from Diamondback Squid (Thysanoteuthis rhombus) Outer Skin," Zeitschrift für Naturforschung, Vol. 59, No. 3-4, 2004, pp. 271-275.

[4] T. Nagai, T. Ogawa, T. Nakamura, T. Ito, H. Nakagawa, K. Fujiki, M. Nakao and T. Yano, "Collagen of Edible Jellyfish Exumbrella," Journal of the Science of Food Agriculture, Vol. 79, No. 6, 1999, pp. 855-858.
[5] T. Nagai, W. Worawattanamateekul, N. Suzuki, T. Nakamura, T. Ito, K. Fujiki, M. Nakao and T. Yano, "Isolation and Characterization of Collagen from Rhizostomous Jellyfish (Rhopilema asamushi)," Food Chemistry, Vol. 70, No. 2, 2000, pp. 205-208.

[6] T. Nagai, E. Yamashita, K. Taniguchi, N. Kanamori and N. Suzuki, "Isolation and Characterization of Collagen from the Outer Skin Waste Material of Cuttlefish (Sepia lycidas)," Food Chemistry, Vol. 72, No. 4, 2001, pp. 425-429.

[7] T. Nagai, Y. Araki and N. Suzuki, "Collagen of the Skin of Ocellate Puffer Fish (Takifugu rubripes)," Food Chemistry, Vol. 78, No. 2, 2002, pp. 173-177.

[8] T. Nagai, K. Nagamori, R. Yamashita and N. Suzuki, "Collagen of Octopus Callistoctopus arakawai Arm," International Journal of Food Science and Technology, Vol. 37, No. 3, 2002, pp. 285-289.

[9] T. Nagai, M. Izumi and M. Ishii, "Fish Scale Collagen. Preparation and Partial Characterization," International Journal of Food Science and Technology, Vol. 39, No. 3, 2004, pp. 239-244.

[10] T. Nagai and N. Suzuki, "Isolation of Collagen from Fish Waste Material-Skin, Bone, and Fins," Food Chemistry, Vol. 68, No. 3, 2000, pp. 277-281.

[11] T. Nagai and N. Suzuki, "Partial Characterization of Collagen from Purple Sea Urchin (Anthocidaris crassispina) Test," International Journal of Food Science and Technology, Vol. 35, No. 5, 2000, pp. 497-501.

[12] T. Nagai and N. Suzuki, "Preparation and Characterization of Several Fish Bone Collagens," Journal of Food Biochemistry, Vol. 24, No. 5, 2000, pp. 427-436.

[13] T. Nagai and N. Suzuki, "Preparation and Partial Characterization of Collagen from Paper Nautilus (Argonauta argo, Linnaeus) Outer Skin," Food Chemistry, Vol. 76, No. 2, 2002, pp. 149-153.

[14] T. Nagai, N. Suzuki and T. Nagashima, "Collagen from Common Minke Whale (Balaenoptera acutorostrata) Unesu," Food Chemistry, Vol. 111, No. 2, 2008, pp. 296-301.

[15] E. Chung and E. J. Miller, "Collagen Polymorphism: Characterization of Molecules with the Chain Composition $[\alpha 1(\mathrm{III})]_{3}$ in Human Tissues," Science, Vol. 183, No. 4130, 1974, pp. 1200-1201.

[16] R. K. Rhodes and E. J. Miller, "Physicochemical Characterization and Molecular Organization of the Collagen A and B Chains," Biochemistry, Vol. 17, No. 1, 1978, pp. 3442-3448.

[17] H. Y. Liu, D. Li and S. D. Guo, "Studies on Collagen from the Skin of Channel Catfish (Ictalurus punctaus)," Food Chemistry, Vol. 101, No. 2, 2007, pp. 621-625.

[18] L. Wang, X. An, Z. Xin, L. Zhao and Q. Hu, "Isolation and Characterization of Collagen from the Skin of Deep-Sea Redfish (Sebastes mentella)," Journal of Food Science, Vol. 72, No. 8, 2007, pp. E450-E455.

[19] Y. Zhang, W. Liu, G. Li, B. Shi, Y. Miao and X. Wu, "Isolation and Partial Characterization of Pepsin-Soluble Collagen from the Skin of Grass Carp (Cteno- 
pharyngodon idella)," Food Chemistry, Vol. 103, No. 4, 2007, pp. 906-912.

[20] S. Kimura, Y. Ohno, Y. Miyauchi and N. Uchida, "Fish Skin Type I Collagen: Wide Distribution of $\alpha 3$ Subunit in Teleosts," Comparative Biochemistry and Physiology, Vol. 88B, No. 2, 1987, pp. 27-34.

[21] S. Kimura and Y. Ohno, "Fish type I collagen: Tissue-Specific Existence of Two Molecular Forms, $(\alpha 1)_{2} \alpha 2$ and $\alpha 1 \alpha 2 \alpha 3$, in Alaska Pollack," Comparative Biochemistry and Physiology, Vol. 88B, No. 2, 1987, pp. 409-413.

[22] B. J. Rigby, "Amino-acid Composition and Thermal Stability of the Skin Collagen of the Antarctic IceFish," Nature, Vol. 219, No. 5150, 1968, pp. 166-167.

[23] M. Ando, M. Ando, M. Makino, Y. Tsukamasa, Y. Makinodan and M. Miyoshi, "Interdependence between Heat Solubility and Pyridinoline Contents of Squid Mantle Collagen," Journal of Food Science, Vol. 66, No. 2, 2001, pp. 265-269.

[24] P. Kittiphattanabawon, S. Benjakul, W. Visessanguan, T. Nagai and N. Tanaka, "Characterisation of AcidSoluble Collagen from Skin and Bone of Bigeye Snapper (Priacanthus tayenus)," Food Chemistry, Vol. 89,
No. 3, 2005, pp. 363-372.

[25] M. Yan, B. Li, X. Zhao, G. Ren, Y. Zhuang, H. Hou, X. Zhang, Li. Chen and Y. Fan, "Characterization of Acid-Soluble Collagen from the Skin of Walleye Pollack (Theragra chalcogramma)," Food Chemistry, Vol. 107, No. 4, 2008, pp. 1581-1586.

[26] B. B. Doyle, E. G. Bendit and E. R. Blout, "Infrared Spectroscopy of Collagen and Collagen-Like Polypeptides," Biopolymers, Vol. 14, No. 5, 1975, pp. 937-957.

[27] K. J. Payne and A. Veis, "Fourier Transform IR Spectroscopy of Collagen and Gelatin Solutions: Deconvolution of the Amide I Band for Conformational Studies," Biopolymers, Vol. 27, No. 11, 1988, pp. 17491760 .

[28] W. K. Surewicz and H. H. Mantsch, "New Insight into Protein Secondary Structure from Resolution Enhanced Infrared Spectra," Biochimica et Biophysica Acta, Vol. 952, No. 2, 1988, pp. 115-130.

[29] R. W. Sarver, Jr and W. C. Krueger, "Protein Secondary Structure from Fourier Transform Infrared Spectroscopy: A Data Base Analysis," Analytical Biochemistry, Vol. 194, No. 1, 1991, pp. 89-100. 